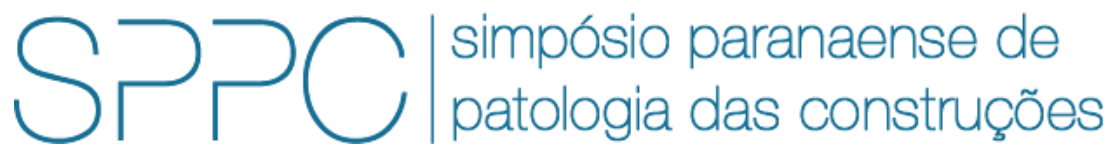

ISSN 2526-7248 artigo 2SPPC1027, pp. 329-343, 2017

\title{
Modelo matemático de ranqueamento - Avaliação da erosão nos pilares de pontes
}

\author{
Cezar Falavigna ${ }^{1}$, André Luiz Tonso Fabiani ${ }^{2}$ \\ ${ }^{1}$ Acadêmico de Engenharia Civil, Universidade Federal do Paraná, cezarfalavigna@gmail.com \\ 2 Professor Doutor, Universidade Federal do Paraná, andre.dhs@ufpr.br
}

Resumo: Ao se conceber uma obra de arte especial (OAE), muitos apectos devem ser levados em consideração em seu projeto, construção e manutenção. O pior cenário para essa estrutura é seu colapso, pois assim ela pode isolar pessoas e áreas, aumentar custos e tempo de viagens, atrasar a entrega de bens e serviços. Uma das maiores causas das quedas de pontes são falhas nos pilares devido aos efeitos de erosão gerados pelo escoamento do rio. Esse enfoque na manutenção dessas estruturas muito vezes não é levado em consideração, por isso é necessário incrementá-la em um modelo de avaliação de obras de arte especiais que já avalie a estrutura como um todo. Esse artigo propõe a análise utilizando o modelo desenvolvido pelo Escritório Modelo de Engenharia Civil (EMEA) da Universidade Federal do Paraná (UFPR)

Palavras-chave: Obras de arte especiais, Pontes, Modelo matemático, Erosão, Erosão nos pilares.

Abstract: From the project, construction and operation of a bridge the worst scenario that can happen is the structure failure. Without the bridge, travels will be longer, costs will be higher, goods and services will be delayed, and even an area can become isolated until it's reconstruction. One of the main reasons of bridge failures are due to pier scour caused by the river the structures are design to transpose. Usually, the bridge maintenance doesn't consider this effect, that's why it is necessary to add this analysis to an evaluation model. This article proposes a way to investigate the scour and how to implement it to the mathematical evaluation model developed by Paraná State Federal University (UFPR) Civil Engineering Model Office (EMEA).

Keywords: Bridges, Scour, Mathematical model, Piers. 


\section{Introdução}

O maior problema que pode ocorre com uma ponte é seu colapso, já que isso pode ocasionar na malha rodoviária ou ferroviária viagens ficarem mais longas, custos mais caros e até mesmo isolar uma região de receber bens e serviços.

Wardhana e Hadipriono [1] estudaram aproximadamente 500 obras que, durante 0 período de 1989 e 2000, ruíram nos Estados Unidos, e percebeu-se que quase 53\% dos colapsos foram por inundações e erosão. Itens como sobrecarga e impacto de embarcações, caminhões ou trens somaram $20 \%$. Chang [2] realizou um estudo envolvendo 383 falhas, dos quais $24,5 \%$ foram relacionados aos pilares e $71,8 \%$ aos encontros, sendo os motivos das quedas divididos em $38,8 \%$ para deficiências no canal, $29,6 \%$ à mudança vigorosa do escoamento, $20,0 \%$ ocasionado por detritos e somente $4,7 \%$ decorrentes de problemas estruturais.

Por esses motivos, analisar a vulnerabilidade das pontes em relação a parte hidráulica precisa ser incrementado em um modelo de avaliação global da estrutura. O modelo matemático escolhido foi o desenvolvido pelo Escritório Modelo de Engenharia Civil - EMEA da Universidade Federal do Paraná foi o escolhido.

\section{Metodologia}

\subsection{Modelo matemático de avaliação - EMEA/UFPR}

O modelo desenvolvido no EMEA/UFPR foi baseado em uma metodoogia aplicada na Eslovênia e Áustria e originalmente descrito no COST 345 [3] do CEB. Ele consiste em ponderar sobre os seguintes parâmetros, dano, elemento estrutural, intensidade do dano, extensão da propagação nos elementos e urgência de reparo conforme ilustra a equação 1 .

$$
R=\sum V_{d}=\sum B_{i} * K_{1 i} * K_{2 i} * K_{3 i} * K_{4 i}
$$

$V_{d}=$ valor do dano;

$\mathrm{Bi}=$ Valor associado ao tipo de dano " $\mathrm{i}$ " sobre a segurança e/ou durabilidade;

$\mathrm{K} 1 \mathrm{i}$ = fator do elemento da estrutura, em função da sua importância no contexto da estrutura como um todo;

$\mathrm{K}_{2 \mathrm{i}}=$ fator indicativo da intensidade do tipo de dano " $\mathrm{i}$ ";

$\mathrm{K}_{3} \mathrm{i}$ = fator relativo à extensão da propagação do tipo de dano " $\mathrm{i}$ " nos elementos inspecionados; e

$\mathrm{K} 4 \mathrm{i}$ = fator enfatizante da urgência de intervenção para o dano " $i$ ".

Segundo o "Bulletin" 243 [4] do CEB, cada um desses índices possue um intervalo de valores nos quais se enquadram, resumido para cada na tabela 1. 
Tabela 1: Intervalo de variação dos parâmetros do modelo EMEA/UFPR

\begin{tabular}{cc}
\hline Parâmetro & Intervalo \\
\hline B - tipo de dano (manifestação patológica) & 1,00 a 5,00 \\
K1 - fator do elemento da estrutura & 0,10 a 0,60 \\
K2 - fator indicativo da intensidade do dano & 0,00 a 2,00 \\
K3 - fator relativo à extensão da propagação do dano & 0,50 a 2,00 \\
K4 - fator enfatizante da urgência da intervenção & 1,00 a 5,00 \\
\hline
\end{tabular}

Entretanto, gerar uma avaliação se limitando apenas aos valores dos índices é errôneo para comparações, pois as pontes possuem diferentes morfologias. Um exemplo, é a comparacação de uma OAE multivigas com uma de viga caixão, a primeira apresenta, no mínimo, mais que duas vigas enquanto a outra apresenta uma, resultando em notas diferentes.

Para não existir esse problema, o resultado dos parâmetros deve ser divido por um valor de referência, assim obtém-se um índice de performance. $O$ valor de referência é atribuído a todos os danos que podem ocorrer na estrutura, multiplicados pelos valores máximos de intenside e extensão e pelo valor unitário de urgência $(K 4=1,00)$. $O$ índice de performance é equacionado a seguir (Eq. 2):

$$
I_{p}=\frac{\sum V_{d}}{\sum V_{d, r e f}}(2)
$$

Ip = índice de performance;

Vd = Valor do dano; e

Vd,ref $=$ valor de referência

Explanado sobre o modelo de avaliação desenvolvido no EMEA/UFPR, parte-se para incrementar a análise hidráulica nesse. Esta é importante ser analisada em três quesitos, o acréscimo de força horizontal nos pilas devido o acúmulo de detritos, se o gabarito é suficiente para o escoamento do rio, e o objetivo deste artigo que é a erosão nos pilares de pontes.

\subsection{Análise da segurança hidráulica no modelo EMEA/UFPR - Erosão nos pilares}

Como citado anteriormente, a avaliação dos quesitos hidráulicos será feita em três partes, porém nesse artigo só será relatado a erosão nos pilares. Inicialmente será descrito como é feita a ponderação da análise e seu embasamento teórico, em seguida, é explanado como aplicar o diagnóstico no modelo matemático do EMEA/UFPR.

Pinto [5] afirma que com a implantação dos pilares ou estacas das pontes, a contração da seção provoca um aumento da velocidade média e da turbulência do escoamento, resultando em uma escavação do leito nas imediações da estrutura. Existem 3 tipos de escavação, erosão do leito, erosão geral ao longo da seção, e o objeto de estudo desse artigo, erosão local. Esta é provocada pelas alterações do movimento junto aos pilares ou encontros da ponte. 
O fenômeno não apresenta maior importância no caso de estruturas assentadas em rocha, porém é comum em rios encontrar material erodível em uma camada espessa o que tornaria economicamente inviável estendê-la até o leito rochoso.

Segundo Tozzi e Ota [6], o perfeito conhecimento do fenômeno de erosão provocado por pilares de ponte exige a compreensão das características hidráulicas do escoamento ao redor do obstáculo.

A natureza do fenômeno, no qual, estão presentes correntes secundárias, zonas de separação, vórtices, e outros, é extremamente complexo e de difícil tratamento matemático. Por isso, torna-se necessário a realização de estudos teóricos e, principalmente, de estudos experimentais para a quantificação do fenômeno.

A principal finalidade de estudar o processo erosivo ao redor de pilares de ponte é obter a previsão máxima da erosão provocada pelas condições de escoamento. A profundidade, devido ao fato de exercer influência na estabilidade estrutural da OAE, possui maior importância e por isso, deve-se correlacionar os parâmetros de escoamento a essa variável. Segundo Ota e Tozzi [6], a profundidade de erosão $\left(\mathrm{ys}_{\mathrm{s}}\right)$ depende de variáveis que caracterizam o fluido $(\rho, v)$, do material do fundo $\left(d, \rho_{s}\right)$, do escoamento (yo, U) e do pilar da ponte (b), conforme ilustrado abaixo:

$$
y_{s}=f\left(\rho, v, g, d, \rho_{s}, y_{o}, U, b\right)(3)
$$

A expressão por ser reescrita admensionalmente como:

$$
\frac{\mathrm{y}_{\mathrm{s}}}{\mathrm{b}}=\mathrm{f}\left(\frac{\mathrm{U} \cdot \mathrm{b}}{v}, \frac{\mathrm{U}^{2}}{\mathrm{~g} \cdot \mathrm{b}}, \frac{\mathrm{y}_{\mathrm{o}}}{\mathrm{b}}, \frac{\mathrm{d}}{\mathrm{b}}, \frac{\rho_{\mathrm{s}}-\rho}{\rho}\right)
$$

Ou ainda:

$$
\frac{y_{s}}{b}=f\left(\frac{v_{*} \cdot b}{v}, \frac{v_{*}^{2}}{g . \delta . b}, \frac{y_{o}}{b}, \frac{d}{b}, \delta_{s}-1\right)
$$

Com $\Delta=\delta s-1$ e $v^{*}=(\text { g.yo.S })^{1 / 2}$, sendo:

$\mathrm{ys}_{\mathrm{s}}=$ profundidade de erosão;

$\mathrm{b}=$ dimensão característica do pilar;

$\mathrm{V}^{\star}=$ velocidade de corte;

$\rho=$ densidade do fluido;

$\mathrm{v}=$ viscosidade cinemática do fluido;

$\mathrm{g}=$ aceleração da gravidade;

$\mathrm{d}$ = dimensão característica do material sólido;

$\rho_{s}=$ densidade do material sólido;

$\mathrm{y}_{\mathrm{o}}=$ profundidade do escoamento;

$U=$ velocidade média do escoamento;

$\delta=$ densidade relativa do material sólido; e 
FALAVIGNA, C.; FABIANI, A. L. T., MODELO MATEMÁTICO DE RANQUEAMENTO - AVALIAÇÃO DA EROSÃO NOS PILARES DE PONTES. $2^{\circ}$ Simpósio Paranaense de Patologia das Construções (20 SPPC), artigo 2SPPC1027, pp. 329-343, 2017. DOI:

$\mathrm{S}=$ declividade da linha de energia

A orientação do pilar é um parâmetro adicional a ser considerado na determinação da profundidade de erosão $\left(y_{s}\right)$. Esta pode ser calculada de duas maneiras conforme o material de fundo, se existir ou não o transporte de partículas. Primeiramente será descrito com a inexistência do transporte.

\subsubsection{Efeito da distribuição da dimensão do sedimento - Ko}

A figura 1 resume os resultados obtidos por Raudkivi [7] para a profundidade máxima de escavação em relação ao diâmetro do pilar ( $\mathrm{y}_{\mathrm{s} / \mathrm{b})}$ como função das características representadas por $\sigma / \mathrm{d}_{50}$, onde $\sigma$ (sigma) é o desvio padrão da distribuição da dimensão do grão e d50, o diâmetro médio da partícula.

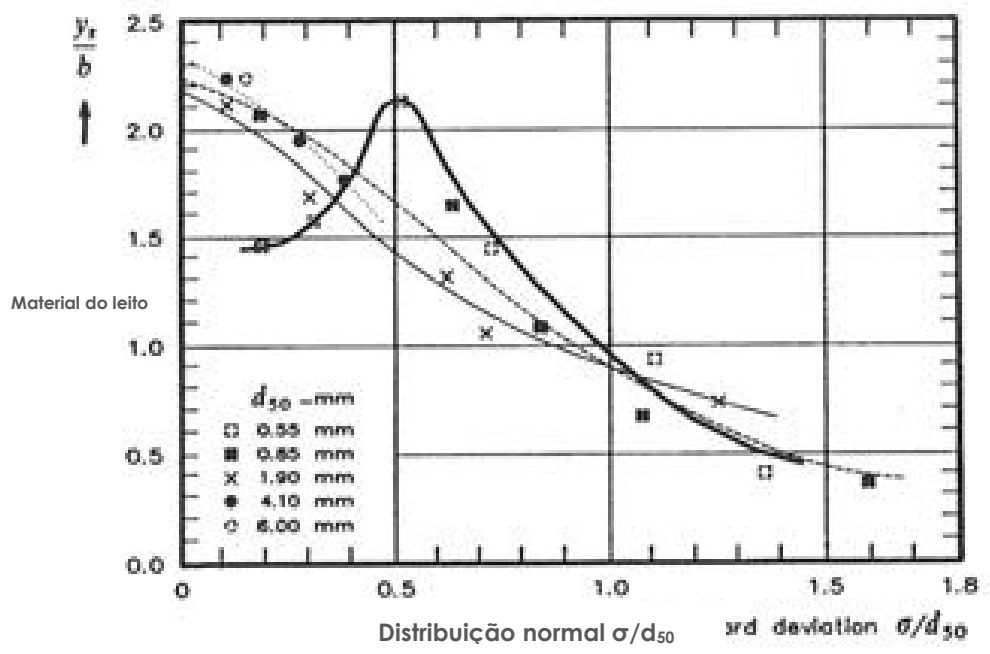

Figura 1: Resultados da profundidade máxima de escavação em relação ao diâmetro do pilar (ys/b). [7]

Percebe-se que a máxima profundidade obtida para material uniforme $(\sigma=0)$, considerando $d>0,7 \mathrm{~mm}$, leva à relação $y_{s} / b=2,1$ a 2,3. Assim, se adota $a$ expressão geral igual a (Eq. 6):

$$
\frac{y_{s}}{b}=2,3(6)
$$

A medida que o desvio padrão aumenta ( $\sigma$ ), os grãos maiores formam uma camada protetora ("armouring") no leito de montante. Existe um valor crítico para $\sigma / \mathrm{d}_{50}$, no qual essa camada pode ser obtida no leito plano, mas não no local onde é escavado, no qual as forças sobre os grãos são maiores decorrente do aumento da agitação turbulenta. Percebe que para areia, cujo $d_{50}=0,55 \mathrm{~mm}$, a profundidade máxima foi igual a 2,1 e aumentando o valor de $\sigma / d_{50}$, o $y_{s} / b$ cai para até 0,3 . Portanto existe influência do desvio padrão (granulometria), que é considerado na abaixo (Eq. 7):

$$
\frac{y_{s}}{b}=2,3 K_{\sigma}(7)
$$


Ko pode ser obtido segundo o gráfico (Fig. 2) a seguir:

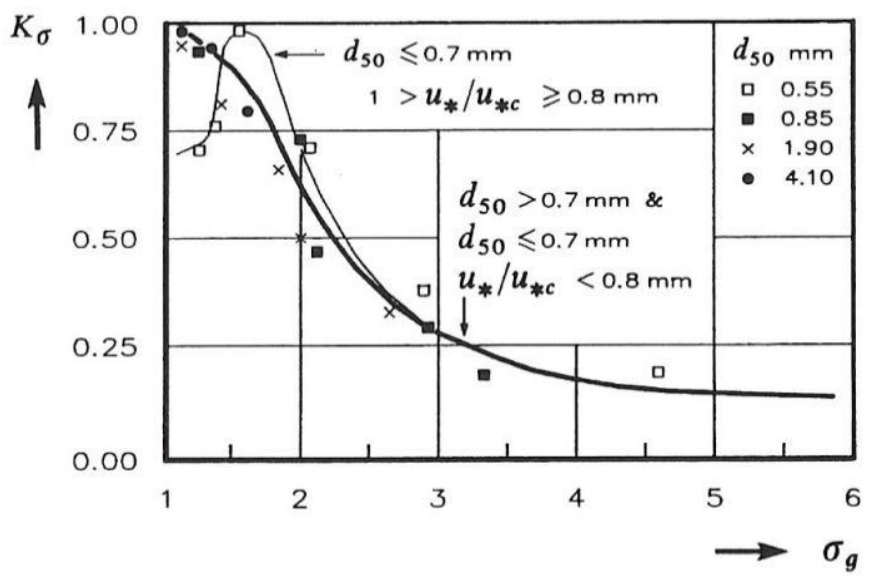

Figura 2: Efeito da distribuição da dimensão do sedimento - Ko. [7]

\subsubsection{Efeito das dimensões do pilar e do sedimento $-K\left(b / d_{50}\right)$}

As dimensões do pilar e do sedimento modificam o desenvolvimento e a profundidade de estabilização da erosão. Por isso, a relação ys/b deve ser corrigida, isso é feito pelo parâmetro $\mathrm{K}\left(\mathrm{b} / \mathrm{d}_{50}\right)$ ilustrado na figura 3 , a seguir.

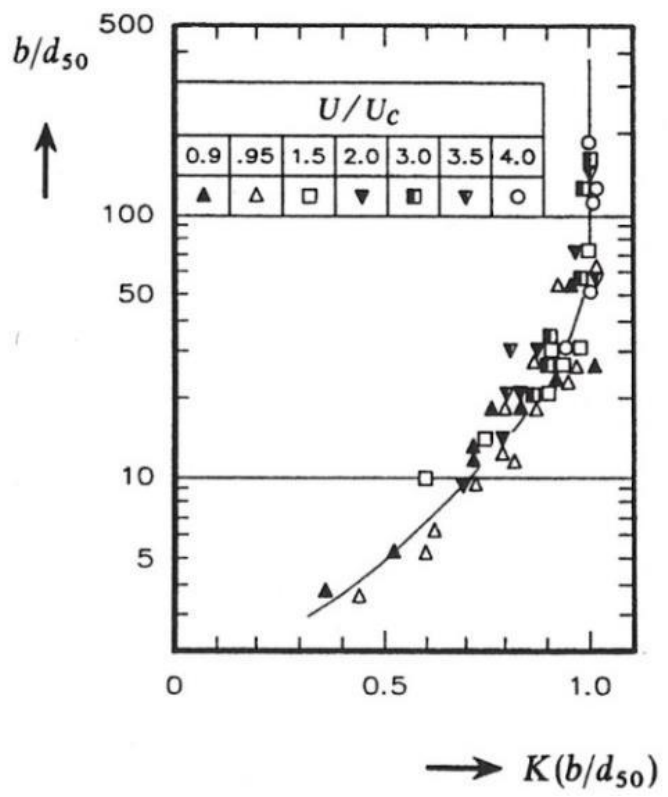

Figura 3: Efeito das dimensões do pilar e do sedimento - K(b/d50). [7]

Os efeitos da erosão são cumulativos, por esse motivo ilustra-se, até agora, como expressão geral (Eq. 8), o seguinte:

$$
\frac{\mathrm{y}_{\mathrm{s}}}{\mathrm{b}}=2,3 \cdot \mathrm{K}_{\sigma} \cdot \mathrm{K}_{\left(\mathrm{b} / \mathrm{d}_{50}\right)}
$$

2.2.3 Efeito da profundidade do escoamento $-K_{d}$ 
Segundo Raudkivi [7], o escoamento tri-dimensional ao longo do pilar impede determinar analiticamente o efeito da profundidade do escoamento (yo) sobre a profundidade da erosão ( $\left.\mathrm{y}_{\mathrm{s}}\right)$. No entanto, Ota e Tozzi [6] ressaltam que observações mostram que:

- Para escoamentos de baixa profundidade, ys cresce com yo;

- Para escoamentos de maior profundidade, ys praticamente independe de yo.

A correção de $y_{s} / b$ devido a esse efeito é feito pelo parâmetro $K_{d}$, obtido pela figura 4 , a seguir. Nota-se que quanto mais fino o sedimento relativamente à dimensão do pilar, menor é a faixa de influência da profundidade do escoamento.

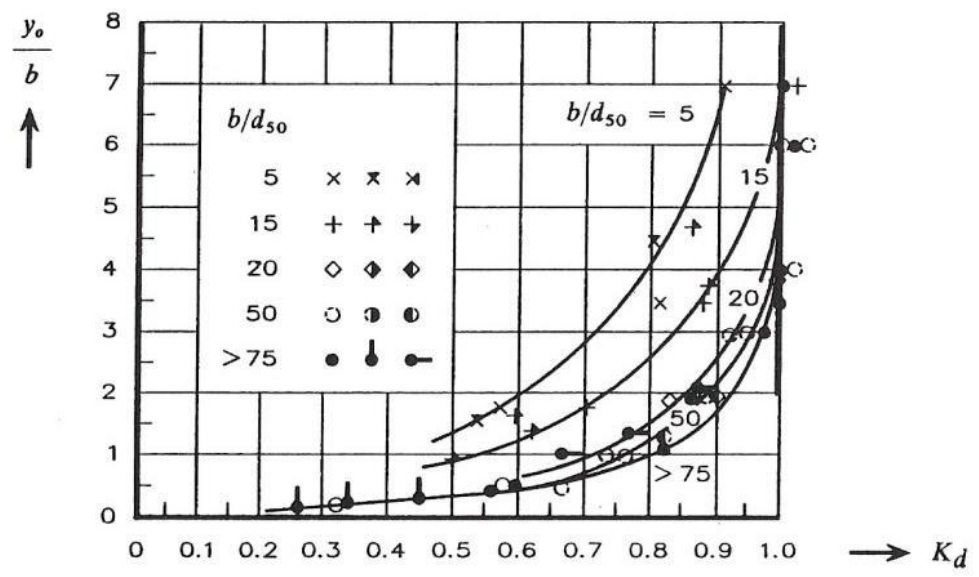

Figura 4: Efeito da profunidade do escoamento - $\mathrm{K}_{\mathrm{d}}$. [7]

\subsubsection{Efeito do alinhamento do pilar - Ka}

O alinhamento ao escoamento da forma do pilar, a exceção dos cilíndricos, altera fortemente a profundidade da erosão, já que esta é função da dimensão normal à corrente. Conforme o ângulo de ataque aumenta, o ponto da máxima profundidade se move ao longo do lado exposto do pilar em direção a sua parte final. $O$ ângulo de ataque depende da relação entre comprimento e da largura do pilar. A figura 5, a seguir, ilustra o parâmetro $K \alpha$, função do ângulo $(\alpha)$ de ataque e relação $\mathrm{I} / \mathrm{b}$ (comprimento/largura), onde Ka irá corrigir o $\mathrm{y}_{\mathrm{s}} / \mathrm{b}$.

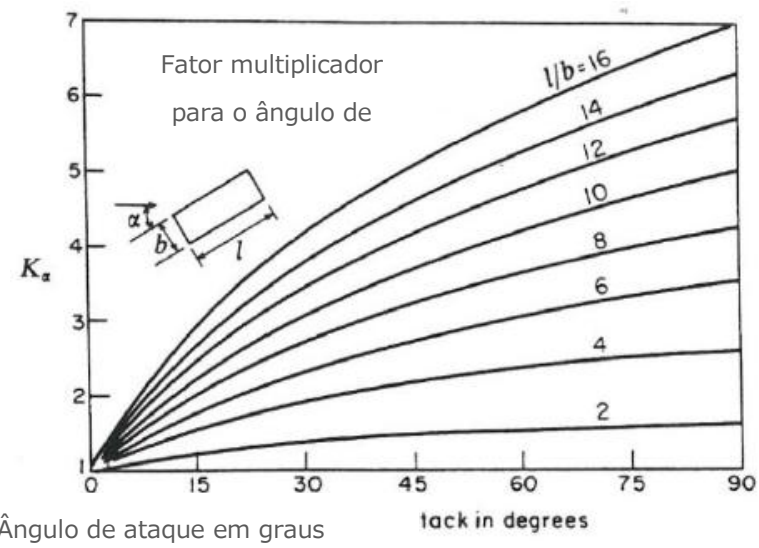

Figura 5: Efeito do alinhamento do pilar - Ka. [7] 


\subsubsection{Efeito da forma do pilar $-K_{s}$}

O efeito da forma do pilar é levado em consideração no Ks. Esse método é aproximado, visto que existe uma enorme variedade de formas de pilar, além do fato que essa forma pode ser alterada pelo acúmulo de material a montante durante enchentes ou trazidos pelo escoamento. Para pilares alinhados (perpendicular ao escoamento) com o escoamento, os valores de $\mathrm{K}_{\mathrm{s}}$ estão disponibilizados no quadro abaixo.

Tabela 2: Efeito da forma do pilar - Ks. [7]

\begin{tabular}{|c|c|c|c|}
\hline Forma do pilar & b/l & $b^{\prime} / l^{\prime}$ & $\mathrm{K}_{\mathbf{s}}$ \\
\hline Cilíndrico & & & 1,0 \\
\hline \multirow[t]{3}{*}{ Retangular } & $1: 1$ & & 1,22 \\
\hline & $1: 3$ & & 1,08 \\
\hline & $1: 5$ & & 0,99 \\
\hline Retangular com face semicircular & & & 0,90 \\
\hline Semicircular com final em cunha & & & 0,86 \\
\hline Retangular com cantos chanfrados & & & 1,01 \\
\hline \multirow[t]{2}{*}{ Retangular com face em cunha } & & $1: 2$ & 0,76 \\
\hline & & $1: 4$ & 0,65 \\
\hline \multirow{3}{*}{ Elípctico } & $1: 2$ & & 0,83 \\
\hline & $1: 3$ & & 0,80 \\
\hline & $1: 5$ & & 0,61 \\
\hline \multirow[t]{2}{*}{ Lenticular } & $1: 2$ & & 0,80 \\
\hline & $1: 3$ & & 0,70 \\
\hline Aerodinâmico & $1: 3,5$ & & 0,80 \\
\hline
\end{tabular}

Portanto, deve-se multiplicar o alinhamento pela forma afim de se o efeito dos pilares sobre a erosão, na equação 9 , a seguir, o alinhamento será admitido como $\mathrm{Ka}$ e a forma como $\mathrm{K}_{\mathrm{s}}$. Com esses parâmetros estabelecidos é possível representar a equação de $y_{s} / b$ por (Eq. 9):

$$
\frac{y_{s}}{b}=2,3 \cdot K_{\sigma} \cdot K_{\left(b / d_{50}\right)} \cdot K_{d} \cdot K_{\alpha} \cdot K_{s}
$$

$y_{s} / b=$ profundidade máxima de erosão;

$\mathrm{K} \sigma$ = coeficiente para o efeito da distribuição da dimensão do sedimento;

$\mathrm{K}(\mathrm{b} / \mathrm{d} 50)=$ coeficiente para o efeito das dimensões do pilar e do sedimento;

$\mathrm{K}_{\mathrm{d}}=$ coeficiente para o efeito da profundidade de escoamento;

$\mathrm{K} \alpha=$ coeficiente para o efeito do alinhamento do pilar; $\mathrm{e}$

$\mathrm{K}_{\mathrm{s}}=$ coeficiente para $\mathrm{o}$ efeito da forma do pilar. 


\subsubsection{Existência de transporte de material do fundo ("Live-Bed Scour")}

Com o transporte de material do fundo, o sedimento que vem de montante atinge 0 buraco da escavação e sai novamente. As características da escavação junto ao pilar para ambos os casos de inexistência (clear water scour) e existência (live-bed scour) de transporte de material do fundo estão descritos na figura 7 , a seguir (considerando o efeito da distribuição granulométrica). $O$ símbolo $U_{c}$ representa a velocidade crítica para o início do transporte do sedimento, ou seja, na figura 6 é ilustrado quantas vezes a mais a velocidade é superior ao início de transporte.

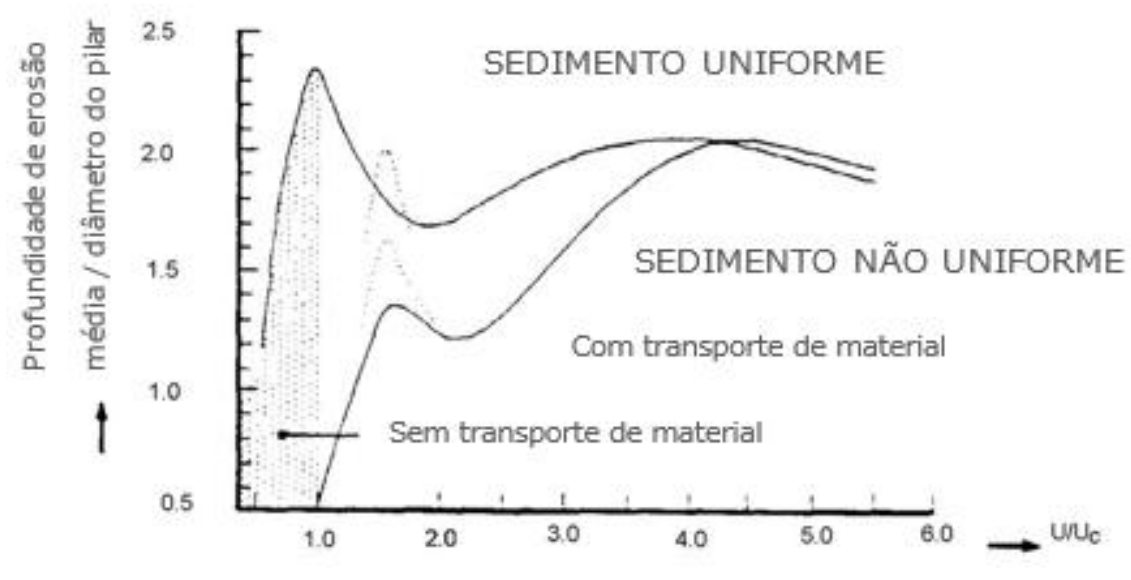

Figura 6: Características da escavação junto ao pilar. [7]

É possível abordar seguintes constatações da figura:

- A curva superior representa o sedimento uniforme, nela verifica-se que a profundidade de erosão decresce do valor de pico conforme a relação U/Uc aumenta, atingindo um valor máximo entre 1,5 e 2,0. Com o aumento de $\mathrm{U} / \mathrm{Uc}$, a profundidade atinge um novo pico denominado "transition flat bed peak";

- O efeito da distribuição do tamanho do sedimento sobre a profundidade de erosão ainda é objeto de estudo. Ota \& Tozzi (2000) [6] ressaltam que uma conclusão simplificadora do processo é que se todos os grãos estão em movimento, a profundidade de erosão no trecho correspondente ao "transition flat bed peak" é pouco afetada. Ela é válida desde que os grãos de maiores dimensões em transporte tenham diâmetros menores que $10 \%$ do diâmetro do pilar e que o escoamento permaneça subcrítico (fluvial). De modo geral, esse efeito parece ser desprezível para og menor que 2 e a profundidade de erosão segue aproximadamente a curva do sedimento uniforme.

Para variações superiores do tamanho do sedimento, a erosão para condição $\mathrm{U} / \mathrm{U}_{\mathrm{c}}$ $=1,0$ é corrigida pelo fator $\mathrm{K} \sigma$ (figura 2), com o valor de $U_{c}$ calculado em função do diâmetro d $d_{50}$.

O início do transporte do sedimento sobre um leito composto de sedimento nãouniforme marca o início do processo de "armouring" da superfície do leito. O "armouring" provoca o aumento do valor da velocidade de corte que a superfície do leito pode resistir e a profundidade local de erosão aumenta até o limite da 
FALAVIGNA, C.; FABIANI, A. L. T., MODELO MATEMÁTICO DE RANQUEAMENTO - AVALIAÇÃO DA EROSÃO NOS PILARES DE PONTES. $2^{\circ}$ Simpósio Paranaense de Patologia das Construções (20 SPPC), artigo 2SPPC1027, pp. 329-343, 2017. DOI:

velocidade de corte $\left(\bigcup^{*} \mathrm{ca}\right)$ ou velocidade média $\left(\bigcup_{\mathrm{ca}}\right)$ da camada de "armour" do sedimento. Para esse valor de Uca, a profundidade de erosão atinge um pico mais baixo, devido ao montante de sedimento em transporte. Ultrapassando esse valor, a camada de superfície do leito é erodida, o transporte de sedimento aumenta rapidamente, conduzindo a uma redução de profundidade de erosão. Na sequência, a profundidade de erosão aumenta novamente com o aumento da tensão tangencial aplicada a condição de "transition flat bed peak". A velocidade crítica sobre uma camada de armouring, $U_{c a}$, pode ser estimada pelo diâmetro $\mathrm{d}_{50}$ dessa camada, denominado d50a. O valor máximo de d50a é:

$$
d_{50 a}=d_{\max } / 1,8
$$

No qual dmax é o diâmetro máximo do material do leito. $O$ valor de d50a permite o cálculo de U*ca pelo critério de Shields (Eq. 10):

$$
\frac{\mathrm{U}_{* \mathrm{ca}}{ }^{2}}{\text { g. } \mathrm{d}_{50 \mathrm{a} \cdot}\left(\delta_{\mathrm{s}}-1\right)}=0,056(10)
$$

A velocidade média crítica correspondente, obtida pela expressão (Eq. 11):

$$
\mathrm{U}_{\mathrm{ca}}=\mathrm{U}_{* \mathrm{ca}}\left[5,75 * \log \left(\frac{\mathrm{y}_{\mathrm{o}}}{2 \cdot \mathrm{d}_{50}}\right)+6\right]
$$

O processo de estimativa da profundidade local de erosão em um pilar de ponte, para os casos de existir ou não o transporte de sedimento, encontra-se resumido, no fluxograma sequente (Fig. 7). 
FALAVIGNA, C.; FABIANI, A. L. T., MODELO MATEMÁTICO DE RANQUEAMENTO - AVALIAÇÃO DA EROSÃO NOS PILARES DE PONTES. $2^{\circ}$ Simpósio Paranaense de Patologia das Construções (20 SPPC), artigo 2SPPC1027, pp. 329-343, 2017. DOI:

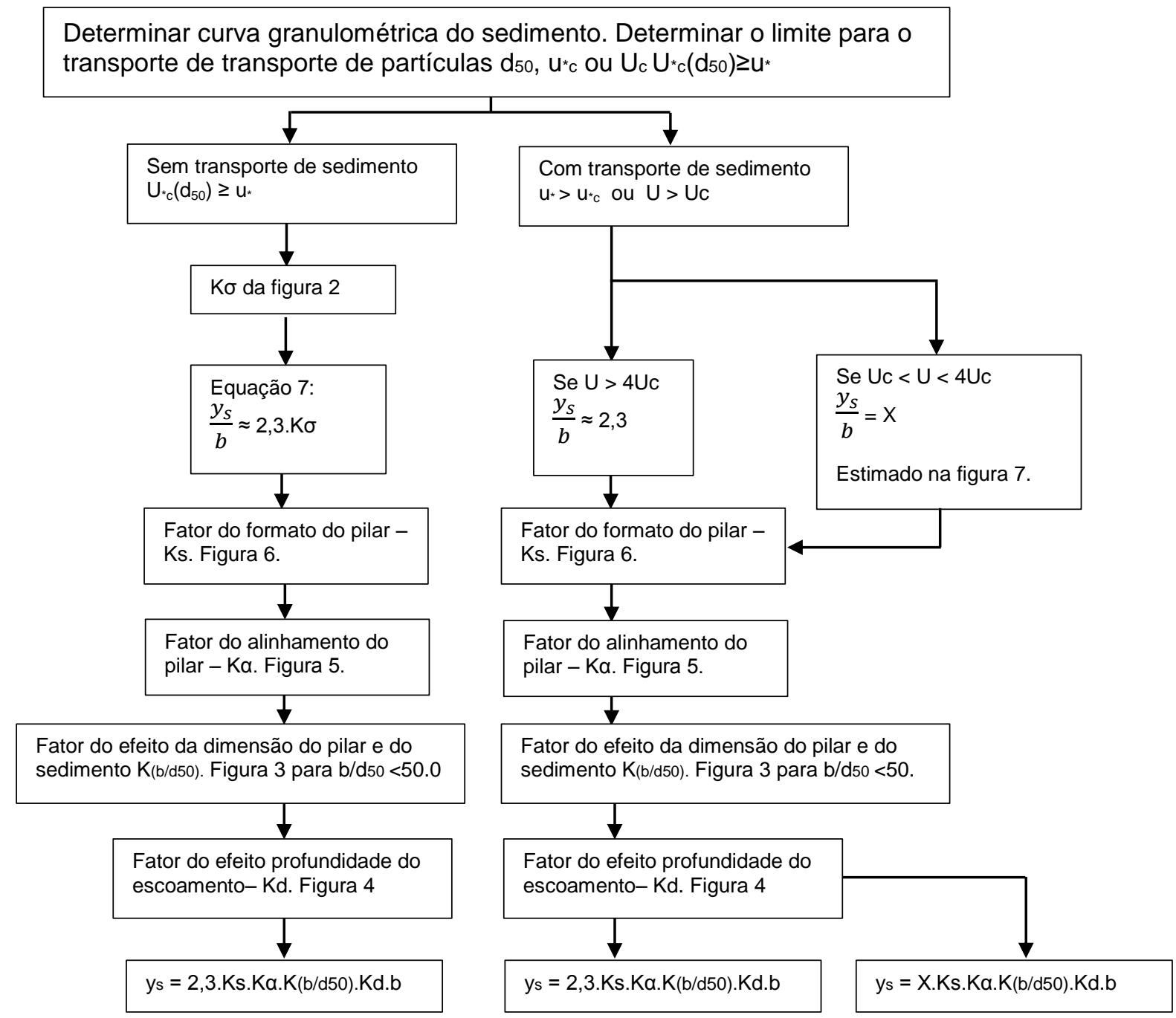

Figura 7: Fluxograma do processo de obtenção da profundidade de erosão. Adaptado [7]

Para inserir no modelo EMEA/UFPR a erosão, inicialmente, deve-se estabelecer um valor para a manifestação patológica, B. Como não existe esse dano na avaliação, o mesmo foi comparado com dois outros similares "Descalçamento" e "Exposição das estacas", que possuem B iguais a 2,00 e a 3,00 respectivamente. Como exposição das estacas é mais danoso e também as estacas podem estar expostas submersas, por isso adotou-se para erosão nos pilares o valor dessa avaria.

Porém, após explicar a análise falta aplicar no modelo do EMEA/UFPR. Inicialemtente, deve ser elencado o valor para o K1, no caso a segurança hídrica, que comparado com outras manifestações do modelo adotou-se 0,40.

Para se estabelecer o K2 deve-se utilizar a seguinte formulação, no qual, é feita a relação entre a profundidade de erosão obtida em fórmula e dividida pela profundidade do canal (Eq. 12). O resultado obtido deve ser comparado com a tabela para obter o K2.

$$
\% \mathrm{~K}_{2}=\left(\frac{\mathrm{y}_{\text {erosão formula }}}{\mathrm{y}_{\text {canal }}}\right) \cdot 100
$$


Tabela 3: Intervalo de valores para o $\mathrm{K} 2$ - Intensidade do dano

\begin{tabular}{cccc}
\hline Tipo & Grau & Critério & Intervalo \\
\hline 0 & Insignificante & Dano irrelevante & 0,00 \\
I & Pequeno & $\begin{array}{c}\text { Dano pequeno, em menos de 10\% da área } \\
\text { total do elemento }\end{array}$ & 0,50 \\
II & Médio & $\begin{array}{c}\text { Dano intermediário, confinado em uma área } \\
\text { pequena de 10\% a 25\% da área total do } \\
\text { elemento }\end{array}$ & 1,00 \\
III & Grande & $\begin{array}{c}\text { Dano grande, de 25\% a 75\% da área total do } \\
\text { elemento, ou em muitos lugares de um } \\
\text { elemento estrutural }\end{array}$ & 1,50 \\
IV & Imenso & $\begin{array}{c}\text { Dano muito grande, na maior parte da área de } \\
\text { um elemento, mais de 75\% da área total do } \\
\text { elemento }\end{array}$ & 2,00 \\
\hline
\end{tabular}

A extensão de propagação do dano, da erosão é obtida pela tabela 4, a seguir:

Tabela 4: Intervalo de valores para o parâmetro K3 - extensão de propagação

\begin{tabular}{cc}
\hline Critério & K3 \\
\hline Dano aparece em menos de 10\% dos elementos estruturais da OAE & 0,50 \\
Dano aparece ente 10\% e 25\% dos elementos estruturais da OAE & 1,00 \\
Dano aparece entre 25\% e 75\% dos elementos estruturais da OAE & 1,50 \\
Dano aparece entre $75 \%$ e $100 \%$ dos elementos estruturais da OAE & 2,00 \\
\hline
\end{tabular}

Como os pilares fora da água não podem sofrer esse tipo de erosão, eles são descartados na análise, sobrando somente os que estão imersos na água. Destes, os que estão próximos das margens possuem menor influência dos que estão no talvegue do rio, isso devido ao fato que próximo das margens as velocidades de escoamento são menores. Por essa razão, esses pilares marginais devem ser minorados. Isso será feito da seguinte maneira (Eq. 13 e 14):

$$
\mathrm{P}=\frac{\mathrm{n}^{\prime} \cdot 100}{\mathrm{n}}
$$

$\mathrm{P}=$ percentual de pilares nas margens do rio;

n' = número de pilares nas margens do rio;

$\mathrm{n}$ = número total de pilares na água.

$$
\mathrm{P}^{\prime}=\frac{\mathrm{P}}{\mathrm{n}^{\prime} \frac{1}{\mathrm{n}^{\prime}}}
$$

$\mathrm{P}^{\prime}=$ percentual minorado dos pilares das margens do rio;

n' = número de pilares nas margens do rio. 
Esse percentual, no entanto, é para todos os pilares, se faz necessário achar um valor individual para os pilares (Eq. 15).

$$
\mathrm{P}_{\text {ind }}^{\prime}=\frac{\mathrm{P}^{\prime}}{\mathrm{n}^{\prime}}
$$

$\mathrm{P}_{\text {ind }}=$ percentual individual do pilar nas margens do rio

$\mathrm{P}^{\prime}=$ percentual minorado do pilar nas margens do rio

n' = número de pilares nas margens do rio

Disso, pode-se calcular o percentual majorado dos outros pilares imersos na região central do rio (Eq. 16) e consequentemente o valor individual desses (Eq. 17).

$$
\mathrm{P}^{\mathrm{a}}=1-\frac{\mathrm{p}}{\mathrm{n}^{\prime} \frac{1}{\mathrm{n}^{\prime}}}
$$

$\mathrm{P}^{\mathrm{a}}=$ percentual majorado dos pilares imersos na região central do rio

$$
\mathrm{P}_{\text {ind }}^{\mathrm{a}}=\frac{\mathrm{P}^{\mathrm{a}}}{\left(\mathrm{n}-\mathrm{n}^{\prime}\right)}
$$

$\mathrm{P}^{\mathrm{a}}=$ percentual majorado individual do pilar imerso na região central do rio

Para a determinação do $\mathrm{K} 3$, basta somar os percentuais dos pilares que estão sofrendo erosão e comparar com a tabela de valores de K3.

$$
\% \mathrm{~K}_{3}=\sum \mathrm{P}_{\text {ind com erosão }}^{\mathrm{a}}
$$

Os valores associados à urgência da intervenção são apresentados abaixo. O dano é classificado de acordo com a tabela 4, a partir da avaliação do inspetor e das condições existentes. Como fatores importantes de análise das condições existentes, recomenda-se ao inspetor observar se a estrutura é isostática ou hiperestática, o comprimento do vão, o número de vãos na sobre a água, se o leito é móvel e se for possível, comparar o perfil do projeto a fim de checar se a profundidade está atingindo a fundação. Este último é o principal, porém nem sempre existe o projeto à disposição para análise.

A partir das informações disponíveis, pode-se atribuir a urgência de reparo conforme a tabela a seguir (Tabela 5): 
FALAVIGNA, C.; FABIANI, A. L. T., MODELO MATEMÁTICO DE RANQUEAMENTO - AVALIAÇÃO DA EROSÃO NOS PILARES DE PONTES. $2^{\circ}$ Simpósio Paranaense de Patologia das Construções (20 SPPC), artigo 2SPPC1027, pp. 329-343, 2017. DOI:

Tabela 5: Intervalo de valores para o parâmetro K4 - urgência de intervenção

\begin{tabular}{ccc}
\hline Classificação & Intervalo & K4 \\
\hline Não urgente & $\begin{array}{c}\text { Intervenção não urgente pois o dano não interfere na } \\
\text { utilização e capacidades da OAE, também não altera a }\end{array}$ & 1,00 \\
Dano à reparar durabilidade & $\begin{array}{c}\text { Dano deve ser reparado em período não maior que 5 } \\
\text { anos, para garantir a servicibilidade e não } \\
\text { compremeter a durabilidade }\end{array}$ & 2,50 \\
Reparo imediato & $\begin{array}{c}\text { Reparo imediato, pois o dano já está comprometendo } \\
\text { a utilização da OAE, com risco às pessoas } \\
\text { Limitação de carga, interrupção de tráfego e }\end{array}$ & 4,00 \\
Limitação de carga & $\begin{array}{c}\text { 5,00 } \\
\text { escoramento imediato deve ser feito, além do reparo }\end{array}$ \\
\hline
\end{tabular}

Com todos os índices estabelecidos, é possível calcular o valor do dano para a erosão nos pilares e assim agregar ao modelo EMEA/UFPR.

\section{Conclusões}

Como citado no início, muitas das falhas que ocorrem em pontes são devido à problemas oriundos do escoamento da água abaixo da estrutura. Então, se faz essencial implementar esse tipo de análise em modelos de avaliação. A erosão, descrita nesse artigo, pode ser facilmente incrementada ao sistema de cálculo do EMEA/UFPR. Com dados que podem ser obtidos em campo, é possível estimar a profundidade de erosão máxima e assim considerar uns dos principais causadores de colapsos em pontes.

A ferramenta descrita no artigo mostra-se aplicável em campo já que não necessita de muitas informações a serem coletadas. Além disso, como na maioria dos casos 0 enfoque de inspeção sempre é limitado a obra e seus parâmetros estruturais, analisar a erosão nos pilares preenche a falta de que quando se inspeciona uma ponte, entretanto, como mencionado anteriormente, os colapsos decorrentes de questões estruturais são bem menos recorrentes do que se comparada às que envolvem o curso d'água. Portanto, o acréscimo nos procedimentos de inspeção, no caso, uma ponderação sobre os efeitos erosivos.

Logo, deve-se somar às inspeções de pontes, avalições sobre o curso d'água onde as pontes estão inseridas, não sobre a pespectiva de um obstáculo a ser transposto e sim como a principal causa de colapsos nessas estruturas.

\section{Agradecimentos}

À Universidade Federal do Paraná, ao Setor de Tecnologia da UFPR, ao Departamento de Construção Civil (DCC) - UFPR, ao Departamento de Hidráulica e Saneamento (DHS) - UFPR, ao Programa de Pós-Graduação em Engenharia de Construção Civil (PPGECC), ao Programa de Pós-Graduação em Engenharia de Recursos Hídricos (PPGERHA), o Escritório Modelo de Engenharia Civil - EMEA da UFPR e principalmente ao professor PhD. Mauro Lacerda Santos Filho por propiciar as condições necessárias para que essa pesquisa fosse realizada. 


\section{Referências}

[1] Wardahana. Kumalasari; Hadripriono, Fabian C. (2003). Analysis of recent bridge failures in the United States. Journal of Performance Of Constructed Facilities. 17:3 p. 144-150.

[2] Chang, F.F.M. (1973). A statistical summary of the cause and cost of bridge failures. Federal Highway Administration - FHWA emergency relief files

[3] European co-operation in the field of scientific and technical research. (2007) .COST 345: Methods used in the european states and assess the condition of highway structures. Disponível em: <http://cost345.zag.si/Reports/COST_345_WG23.pdf>. Acesso em 21 ago. 2016

[4] Comité euro-internacional du betón. (1998). Bulletin 243: Strategies for Testing and Assessment of Concrete Structures. Suíça.

[5] Pinto, N. L. S. (1961). Erosão ao redor de pilares de ponte. 1 v. Tese (Doutorado) - Curso de Engenharia Civil, Universidade Federal do Paraná, Curitiba, Brasil.

[6] Tozzi, M.; OTA; J. J. (2000). Erosão em pilares de pontes.

[7] Raudikivi, A. J. (1991). Scour at bridge piers. In BREUSERS, H. N. C.; RAUDIKIVI, A. J.. Scouring. A.A. Balkema: Rotterdam, p. 61-94. 